\title{
Attraction Domain estimates combining Lyapunov Functions
}

\author{
Donatello Materassi and Murti V. Salapaka
}

\begin{abstract}
This article tackles the problem of estimating the domain of attraction of a Lur'e system, that is the feedback interconnection of a linear time-invariant system with a memoryless static operator. When the dimension of the system is large, numerical approaches based on simulations become prohibitive from a computational point of view. On the other hand, classical analytical techniques based on Lyapunov functions provide conservative estimates because they usually consider quadratic Lyapunov functions. Another limit is given by the fact that they deal with contractively invariant sets, which are sets where the derivative of the Lyapunov function along the trajectories is negative. Methods to reduce their conservativeness are still a challenging subject of research and desirable for many practical applications. In this paper, we try to combine the information given by more Lyapunov functions together in order to enlarge the estimate of the domain of attraction. The novelty of our approach lies in the fact that the sets we are considering are invariant but not necessarily contractively invariant. We assume that an estimate of the attraction domain is already known. In order to show that a set is part of the attraction domain, it is sufficient to prove that all the trajectories starting from it reach the current estimate in a finite time. This concept provides a method to iteratively improve the attraction domain estimate using different Lyapunov functions without limiting the analysis to contractively invariant sets. After developing a general theory, we only resort to the use of quadratic Lyapunov functions because of the computational appeal given by LMI solvers.
\end{abstract}

\section{INTRODUCTION}

In this work, we focus on an important class of nonlinear models given by the feedback interconnection of a linear time-invariant system $\mathcal{G}$ with a nonlinear block $\mathcal{N}$ (see Figure 1). Models of this kind are known in the literature

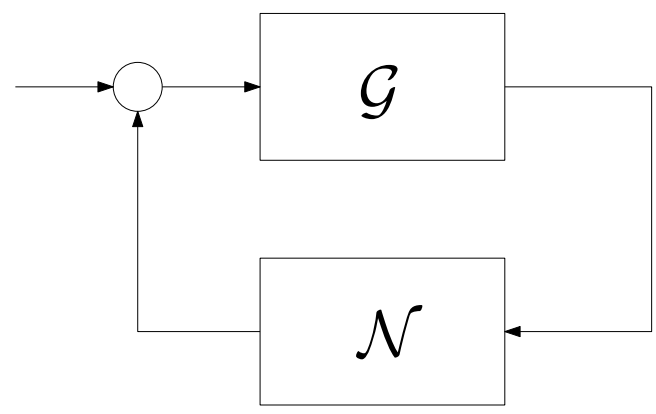

Fig. 1. A Lur'e system

D. Materassi is with Department of Electrical and Computer Engineering, University of Minnesota, 200 Union St SE, 55455, Minneapolis (MN) mater013@umn.edu

M. V. Salapaka is with Department of Electrical and Computer Engineering, University of Minnesota, 200 Union St SE, 55455, Minneapolis (MN) murtis@umn.edu as Lur'e systems [1] and a large number of real systems have this structure. Recent examples are provided by Atomic Force Microscopes [2],[3] and other Microelectromechanical systems [4], [5], [6]. Many studies targeted the problem of "absolute stability", where the global asymptotic stability of the origin is sought with respect to a class of nonlinear operators, and thus providing a robust notion of stability. Classical results include the Popov (see [7]) and the circle criteria (see [8] and [9]) that provide sufficient conditions for global asymptotic stability when the nonlinearity is restricted to be time-invariant and time varying respectively. A relatively new approach involving only input-output maps is given by the Integral Quadratic Constraint (IQC) methods pioneered in [10]. In this approach, a quadratic constraint is used to characterize the nonlinearity playing the role of the sector condition used in the other classical absolute stability criteria. Since the sector relations can be derived as special IQC conditions, such an approach provides a powerful unifying theoretical framework. Furthermore, it provides the powerful capability of seamlessly integrating different characterizations of the nonlinearity, as well. When an equilibrium is not globally asymptotically stable, it is important for many practical issues to estimate its domain of attraction. Indeed, the domain of attraction represents the region of perturbations on the equilibrium which the system can absorb. Estimating a domain of attraction is a difficult problem to solve even from a numerical point of view, especially when the phase space has a large dimension. Any approach based on simulation techniques would require a large number of systematic simulations with different initial conditions. An analytical, but more conservative method to estimate the domain of attraction is provided by the LaSalle theorem [11], [12] which relies on the knowledge of a Lyapunov function. Since both the circle and the Popov criteria rely on the construction of a Lyapunov function in order to prove global asymptotic stability, the same criteria can be suitably modified in order to obtain local results assuming that the nonlinearity satisfies local properties only [11]. Consequentely, an estimate of the attraction domain can be accomplished exploiting the Lyapunov function provided by these criteria in combination with the LaSalle theorem. In order to reduce the conservativeness of this approach, in [13] an optimization technique based on LMI's is proposed. It is based on the computation of the largest contractively invariant ellipsoid of a fixed shape containing the equilibrium. We remind that an ellipsoid is contractively invariant if the derivative of its related quadratic form is negative along the system trajectories. The authors also prove 
that the convex hull of contractively invariant ellipsoids is an invariant contractive set contained in the domain of attraction.

In this paper, we propose a technique to improve the estimate of attraction domains. LMI's are a powerful tool to obtain a quadratic Lyapunov function which proves local stability of an equilibrium. However, in some situations, contractively invariant ellipsoids and their convex hull provide a conservative estimate of the attraction domain. This is due to the strong requirement of "contractive invariance": the ellipsoid must be an invariant set and also the derivative of the Lyapunov function along the contained trajectories must be strictly negative. Conversely, we assume that an estimate of the attraction domain is already known. In order to show that a set is part of the attraction domain, it is sufficient to prove that all the trajectories starting from it reach the current estimate in a finite time. This concept provides a method to iteratively improve the attraction domain estimate without limiting the analysis to contractively invariant sets. In the paper we formalize this intuitive idea developing adequate theoretical tools in order to handle positively invariant sets. After developing a general theory, we only resort to the use of quadratic Lyapunov functions because of the computational appeal given by LMI solvers. We also introduce the concept of Biased Local Quadratic Constraint as a technical instrument to describe a nonlinearity. It is a general formulation of many quadratic constraints which are used to express conditions in many stability criteria (Circle, Popov, Zames-Falb). A more general relaxation of the Integral Quadratic Constraints (IQC's) introduced by [10] could have been pursued. However this is beyond the main objectives of this short article. The paper is structured as follows: in Section III the problem is formulated; in Section IV some preliminary results are illustrated; Section V contains the general theoretical results; Section VI combines the theory with LMI's and BLQC's as practical tools to construct Lyapunov functions; finally Section VII shows the utility and limits of our techniques through two numerical examples, providing also a comparison with other techniques in the literature.

\section{Definitions and Notation}

Definition 1: Given a space $X$ with a metric $d$, we extend the definition of the metric to include the concept of distance between a point $x \in X$ and a set $A \subset X$ in the following way

$$
d(x, A):=\inf \{d(x, y) \mid y \in A\} .
$$

Definition 2: Given a metric space $X$ with a metric $d$ and a set $A \subseteq X$, we define the neighborhood of $A$ with radius $\epsilon$ as

$$
\mathcal{I}(A, \epsilon):=\{x \in X \mid d(x, A)<\epsilon\} .
$$

Consider a dynamical system $\mathcal{S}$ described by an initial condition problem

$$
\begin{aligned}
& \dot{x}=f(t, x) \\
& x\left(t_{0}\right)=x_{0}
\end{aligned}
$$

with $f: \mathfrak{R} \times \mathfrak{R}^{n} \rightarrow \mathfrak{R}^{n}$ "regular enough" in order to guarantee the uniqueness of solutions. Let $x(t):=\phi\left(t, t_{0}, x_{0}\right)$ be the trajectory related to the initial condition $\left(t_{0}, x_{0}\right)$.

Definition 3: We say that $A$ is an Positively Invariant (PI) set for $\mathcal{S}$ if and only if, for all $t>t_{0}$ and for all $x_{0} \in A$

$$
\phi\left(t, t_{0}, x_{0}\right) \in A \quad \text { for all } t>T .
$$

Definition 4: We say that $A$ is an attracting set for $\mathcal{S}$ if, for any $\epsilon>0$, there exist a $\delta=\delta(\epsilon)$ and a $T=T(\epsilon)$ such that

$x\left(t_{0}\right) \in \mathcal{I}(A, \delta)$ implies $x(t) \in \mathcal{I}(A, \epsilon)$ for all $t>T$.

Definition 5: We say that $A$ is an attractor if it is an attracting set that does not contain any other proper attracting subset.

Definition 6: Given an attracting set $A$, we define its domain of attraction as

$$
\begin{aligned}
& \mathcal{D}(A):=\left\{x_{0} \in \mathfrak{R}^{n} \mid\right. \\
& \left.\forall \epsilon>0 \exists T=T\left(\epsilon, x_{0}\right), t>T \text { implies } \phi\left(t, t_{0}, x_{0}\right) \in \mathcal{I}(A, \epsilon)\right\}
\end{aligned}
$$

Definition 7: Let $P$ be a strictly positive $n \times n$ matrix and let $H$ be a real nonnegative value. We define

$$
\mathcal{E}_{P}(H):=\left\{x \in \mathbb{R}^{n}: x^{T} P x \leq H\right\} .
$$

Definition 8: Given a scalar function $V: \mathfrak{R}^{n} \rightarrow \mathfrak{R}$ we define

$$
\Omega_{V}(H):=\left\{x \in \mathbb{R}^{n}: V(x) \leq H\right\} .
$$

Definition 9: Given a constant matrix $\Sigma \geq 0$, we define the quadratic form $\sigma: \mathbb{R}^{n} \times \mathbb{R}^{n} \rightarrow \mathbb{R}$

$$
\sigma(y, u)=\left(\begin{array}{l}
y \\
u
\end{array}\right)^{*} \Sigma\left(\begin{array}{l}
y \\
u
\end{array}\right) .
$$

We say that a nonlinearity $\Xi(\cdot)$ satisfies the Biased Local Quadratic Constraint (BLQC) defined by $\sigma$ with bias $M>0$, if and only if

$$
\sigma(y(t), \xi(t))+M \geq 0 \quad \text { for all } t>0
$$

for any signals $y(t), \xi(t) \in L_{2 e}$, where $\xi(t)=\Xi(y(t))$. We also use the notation $\Xi \in B L Q C(\sigma, M)$ and say that the matrix $\Sigma$ is the multiplier of the BLQC.

\section{PRoblem DEFINITION}

Let us consider a Lur'e system as depicted in Figure 1. Assume that the linear system $\mathcal{G}$ is proper with transfer function $G(s)$ and that the nonlinear block $\mathcal{N}$ can be described by a possibly time-varying operator $\Xi$. The differential equation representing such a feedback interconnection is symbolically expressed by

$$
y=G(d / d t) \Xi(t, y(\cdot))
$$

where $y$ is the output of the system. Assume that the origin is a stable equilibrium for the system. The problem we are considering is to find an estimate of its attraction domain. 


\section{Preliminary Results}

This section introduces some technical results needed to derive the main contributions of the paper. The following lemma provides a bound for the scalar output $y=C x$ of a dynamical system when the state $x$ belongs to the ellipsoid $\mathcal{E}_{P}(H)$.

Lemma 4.1: Let us consider a positive symmetric matrix $P$, then it holds that

$$
\max _{x \in \mathcal{E}_{P}(H)}|C x|=\sqrt{H C P^{-1} C^{T}}
$$

Proof: The lemma is a trivial result of projective geometry.

The following lemma is a technical result which will be exploited in the following section.

Lemma 4.2: Assume that $V: \mathfrak{R}^{n} \rightarrow \mathfrak{R}$ is a continuous function and that $\Omega_{V}(H)$ is a compact set for any $H$. Then, for any $\epsilon>0$ there exists $H_{\epsilon}>H$ such that $x \in \Omega_{V}\left(H_{\epsilon}\right)$ implies that

$$
d\left(x, \Omega_{V}\left(H_{\epsilon}\right)\right)<\epsilon
$$

Proof: By contradiction there exists $\epsilon>0$ such that for any $H_{\epsilon}>H$ it is possible to find a $x \in \Omega_{V}\left(H_{\epsilon}\right)$ with $d\left(x, \Omega_{V}\left(H_{\epsilon}\right)\right) \geq \epsilon$. Consider the sequence $H_{n}=H+1 / n$ and the related sequence $x_{n}$ such that

$$
d\left(x, \Omega_{V}(H)\right) \geq \epsilon
$$

Since $\Omega_{V}(H+1)$ is a compact set, there exists a subsequence $x_{k_{n}}$ converging to $\hat{x} \in \Omega_{V}(H+1)$. By the continuity of $V$ we have that

$$
V(\hat{x})=\lim _{n \rightarrow+\infty} V\left(x_{k_{n}}\right) \leq \lim _{n \rightarrow+\infty}\left(H+\frac{1}{k_{n}}\right)=H
$$

implying that $\hat{x} \in \Omega_{V}(H)$ On the other hand we have

$$
0=d(\hat{x}, \hat{x})=\lim _{n \rightarrow+\infty} d\left(x_{k_{n}}, \hat{x}\right) \geq \liminf _{n \rightarrow+\infty} d\left(x_{k_{n}}, \Omega_{V}(H)\right) \geq \epsilon>0
$$

which is a contradiction.

\section{Main Theoretical Results}

The following theorem employs a scalar function $V$, defined on the state space, in order to detect positively invariant and attracting sets giving an estimate of their domain of attraction.

Theorem 5.1: Consider a dynamical system as in (3) and a scalar function $V: \mathfrak{R}^{n} \rightarrow \mathfrak{R}, V \in C^{1}(\mathfrak{R})$. Assume that

$$
x(t) \in \Omega_{V}(\bar{H}) \backslash \Omega_{V}(\underline{H}) \text { implies } \dot{V}(x(t))<0
$$

for some $\bar{H}>\underline{H}$ and that $\Omega_{V}(H)$ is compact for every $\underline{H}<$ $H<\bar{H}$. Then

- $\Omega_{V}(H)$ is positively invariant

- $\Omega_{V}(\underline{H})$ is an attracting set

- $\Omega_{V}(H) \subseteq \mathcal{D}\left(\Omega_{V}(\underline{H})\right)$.

Proof: First, let us prove that $\Omega_{V}(H)$ is positively invariant. Fix $\epsilon>0$ and consider $x_{0} \in \Omega_{V}(H)$. By contradiction, there exists $t_{3}$ such that $H<V\left(x\left(t_{3}\right)\right):=H^{*}<\bar{H}$. Consider

$$
\begin{aligned}
& t_{1}:=\sup \left\{t \mid 0<t<t_{3}, V(x(t)) \leq H\right\} \\
& t_{2}:=\inf \left\{t \mid t_{1}<t<t_{3}, V(x(t)) \geq H^{*}\right\} \neq t_{1} .
\end{aligned}
$$

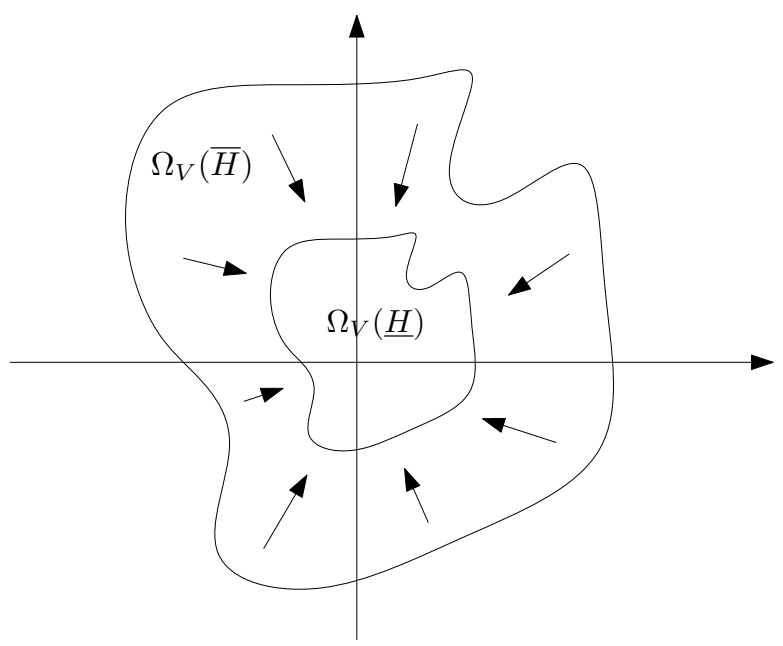

Fig. 2. An intuitive representation of a "contractive shell" in the phase space

Since $V(x(t))$ is a continuous function the sets are not empty and the definitions make sense. Note also that $t_{1}<t<t_{2}$ implies $H<V(x(t))<H^{*}$. By the mean value theorem, we have that

$$
0<V\left(x\left(t_{2}\right)\right)-V\left(x\left(t_{1}\right)\right)=\int_{t_{1}}^{t_{2}} \dot{V}(x(t)) d t=\dot{V}(x(\hat{t}))\left(t_{2}-t_{1}\right)
$$

for some $t_{1}<\hat{t}<t_{2}$, but this is a contradiction since $\dot{V}(x(\hat{t}))<$ 0 .

Now, let us prove that any trajectory with $x_{0} \in \Omega_{V}(H)$ is attracted to $\Omega_{V}(\underline{H})$. Given $\epsilon>0$, it is always possible to find $H_{\epsilon}>\underline{H}$ such that

$$
x \in \Omega_{V}\left(H_{\epsilon}\right) \Rightarrow d\left(x, \Omega_{V}(\underline{H})\right)<\epsilon .
$$

Given $x_{0} \in \Omega_{V}(H)$, there exists $t>0$ such that $x(t) \in \Omega_{V}\left(H_{\epsilon}\right)$. Assume again by contradiction that it does not exist. This means that $V(x(t))>H_{\epsilon}$ for all $t$. Then we have

$$
d V / d t \leq-r:=\min \left\{\dot{V}(x(t)) \mid H_{\epsilon} \leq V(x(t)) \leq H\right\}<0 .
$$

Integrating both sides, we find a contradiction, since $V(x(t))$ should diverge to $-\infty$. Therefore there exists $t>0$ such that $x(t) \in \Omega_{V}\left(H_{\epsilon}\right)$. The fact that $\mathcal{E}_{P}(\underline{H})$ is an attracting set follows from the positively invariance of $\mathcal{E}_{P}\left(H_{\epsilon}\right)$.

The most important property required from $V$ is to have a negative time derivative on the set $\Omega_{V}(\bar{H}) \backslash \Omega_{V}(\underline{H})$ which can be interpreted as a "shell" in the phase space. Then, Theorem 5.1 intuitively states that such a shell is "contractive" in the sense that all trajectories in it approach its inner surface $\{x: V(x)=\underline{H}\}$. A graphical representation of this intuition is given in Figure 2. Note that Theorem 5.1 also provides a generalization of the classical Lyapunov theorem for asymtpotical stability when $\underline{H}=0, V(x)>0$ for any $x \neq 0$.

The following theorem provides a method to include level sets described by a scalar function $V_{2}$ into the attraction domain of attracting sets described by a different scalar function $V_{1}$. 


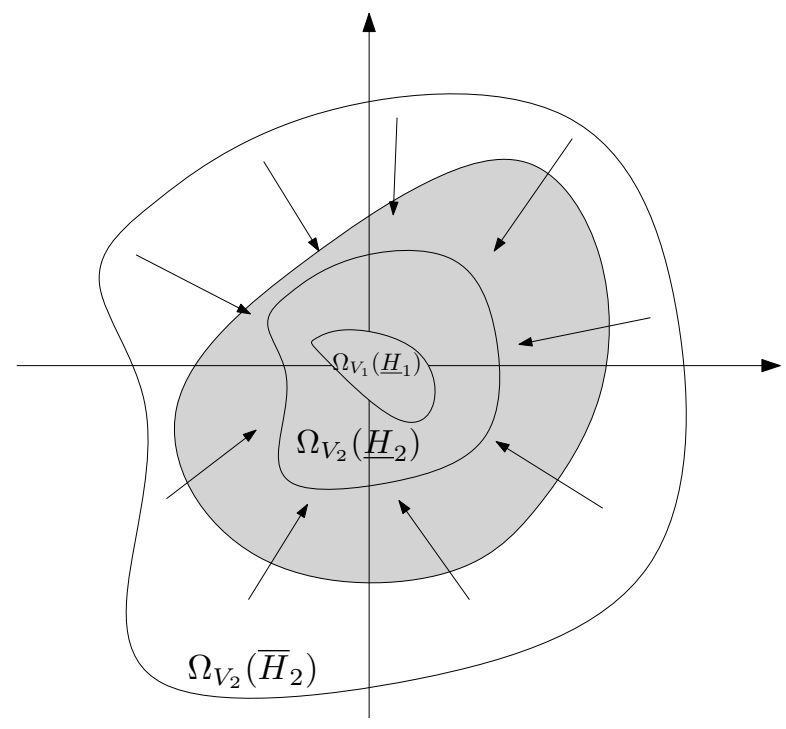

Fig. 3. An intuitive phase space representation of Theorem 5.2. The gray area represents the region where $\dot{V}_{1}$ is negative

Theorem 5.2: Consider a dynamical system as in (3) and two scalar functions $V_{1}, V_{2}: \mathfrak{R}^{n} \rightarrow \mathfrak{R}, V_{i} \in C^{1}(\mathfrak{R})$. Assume that

- $x(t) \in \Omega_{V_{2}}\left(\bar{H}_{2}\right) \backslash \Omega_{V_{2}}\left(\underline{H}_{2}\right)$ implies $\dot{V}_{2}(x(t))<0$

- $x(t) \in \Omega_{V_{2}}\left(\underline{H}_{2}\right) \backslash \Omega_{V_{1}}\left(\underline{H}_{1}\right)$ implies $\dot{V}_{1}(x(t))<0$

- $\Omega_{V_{1}}\left(\underline{H}_{1}\right)$ is an attracting set

for some $\bar{H}_{2}>\underline{H}_{2}, \underline{H}_{1}$. Then

$$
\Omega_{V_{2}}\left(H_{2}\right) \subseteq \mathcal{D}\left(\Omega_{V_{1}}\left(\underline{H}_{1}\right)\right) .
$$

Proof: Given $\mathrm{H}_{2}$ and $\epsilon_{2}>0$, from Lemma 5.1 we know that any solution $x(t)$ with initial condition in $\Omega_{V_{2}}\left(H_{2}\right)$ reaches the positively invariant set $\Omega_{V_{2}}\left(\underline{H}_{2}+\epsilon_{2}\right)$ at a time $t_{1}$. By the continuity of $\dot{V}_{1}(x(t))$ and Lemma 4.2 it is possible to choose $\epsilon_{2}$ small enough, such that $\dot{V}_{1}(x(t))<0$ for any $x(t) \in \Omega_{V_{2}}\left(\underline{H}_{2}+\epsilon_{2}\right) \backslash \Omega_{V_{1}}\left(\underline{H}_{1}\right)$. Now let us prove that $x(t)$ is attracted by $\Omega_{V_{1}}\left(\underline{H}_{1}\right)$. By contradiction, assume that $x(t)$ is not attracted by $\Omega_{V_{1}}\left(\underline{H}_{1}\right)$. Thus, there is a $\epsilon_{1}>0$ such that $d\left(x(t), \Omega_{V_{1}}\left(\underline{H}_{1}\right)\right) \geq \epsilon_{1}$ for any $t$ implying that $V(x(t))>\underline{H}_{1}$ for any $t$. Since $\Omega_{V_{2}}\left(\underline{H}_{2}+\epsilon_{2}\right)$ is a positively invariant set we have that $\dot{V}(x(t))<0$ for any $t>t_{1}$. Consider the relation

$$
\begin{aligned}
& V(x(t))-V\left(x\left(t_{1}\right)\right)= \\
& =\int_{t_{1}}^{t} \dot{V}(x(t)) d t=\dot{V}(x(\hat{t}))\left(t-t_{1}\right)<\eta\left(t-t_{1}\right) .
\end{aligned}
$$

This is a contradiction because it implies that $V(x(t))$ diverges to $-\infty$.

Theorem 5.2 provides a method to enlarge the attraction domain estimate of the attracting set $\Omega_{V_{1}}\left(\underline{H}_{1}\right)$ using level sets of the different Lyapunov function $V_{2}$. This is possible, according to the theorem hypothesis, when the contractive shell $\Omega_{V_{2}}\left(\bar{H}_{2}\right) \backslash \Omega_{V_{2}}\left(\underline{H}_{2}\right)$ has its inner surface contained in a region where $\dot{V}_{1}$ is negative. An intuitive graphical representation is provided in Figure 3. It is important to stress that the set $\Omega_{V_{2}}\left(\bar{H}_{2}\right)$ is not required to be contractively invariant in order to be added to the estimate of attraction domain.

\section{LMI's AND BLQC's TO ESTIMATE DOMAINS OF ATTRACTION}

In this section we exploit BLQC's and LMI's as practical tools to find attracting sets and estimate their domains. We show the following results.

Theorem 6.1: Let $\mathcal{S}$ be the Lur'e system described by (6). Let $G(s)$ be a strictly proper transfer function and let $(A, B, C, 0)$ be a minimal realization of $G(s)$ with state $x \in \mathbb{R}^{n}$. Suppose that, for any $y$ such that $y^{2}<Y^{2}, \Xi$ satisfies the BLQC given by the quadratic form $\sigma$

$$
\sigma(y, \xi)=\left(\begin{array}{c}
y \\
\xi
\end{array}\right)^{T}\left(\begin{array}{cc}
Q & S \\
S^{T} & R .
\end{array}\right)\left(\begin{array}{c}
y \\
\xi
\end{array}\right) \geq-M .
$$

Assume that there exists a solution $(P, r)$ to the following LMI

$$
\left[\begin{array}{cc}
A^{T} P+P A+C^{T} Q C+r P & P B+C^{T} S \\
B^{T} P+S^{T} C & R
\end{array}\right]<0
$$

with $P \in \mathbb{R}^{n \times n}, P=P^{T}, P>0, r \in \mathbb{R}^{+}$and define

$$
\underline{H}:=\frac{M}{r} \quad \bar{H}:=\frac{Y^{2}}{C P^{-1} C^{T}} .
$$

If there exists $H$ such that $\underline{H}<H<\bar{H}$, then the set $\mathcal{E}_{P}(H)$ is a positively invariant set for $\mathcal{S}$. Moreover $\mathcal{E}_{P}(\underline{H})$ is an attracting set and $\mathcal{E}_{P}(H)$ is contained in its attraction domain.

Proof: Define the Lyapunov function

$$
V(x)=x^{T} P x .
$$

The derivative of $V(x(t))$ along any trajectory satisfies

$$
\begin{aligned}
d V / d t= & x^{T} A^{T} P x+x^{T} P A x+\xi^{T} B^{T} P x+x^{T} P B \xi= \\
= & x^{T} A^{T} P x+x^{T} P A x+\xi^{T} B^{T} P x+x^{T} P B \xi+ \\
& +x^{T} C^{T} Q C x+2 x^{T} C^{T} S \xi+\xi^{T} R \xi-\sigma(y, \xi) \leq \\
\leq & -r x^{T} P x-\sigma(y, \xi)
\end{aligned}
$$

Observe that the hypothesis of Theorem 5.1 are satisfied, so the assertion is proven.

The following theorem is the main contribution of the paper providing an iterative technique to enlarge the estimate of a domain of attraction using $N$ different scalar functions.

Theorem 6.2: Let $\mathcal{S}$ be the Lur'e system described by (6). Let $G(s)$ be a strictly proper transfer function and let $(A, B, C, 0)$ be a minimal realization of $G(s)$ with state $x \in$ $\mathbb{R}^{n}$. Suppose that, for $i \in\{1,2, \ldots, N\}, y(t)^{2}<Y_{i}^{2}$ implies $\Xi \in B L Q C\left(\sigma_{i}, M_{i}\right)$ where

$$
\sigma_{i}(y, \xi)=\left(\begin{array}{c}
y \\
\xi
\end{array}\right)^{T}\left(\begin{array}{cc}
Q_{i} & S_{i} \\
S_{i}^{T} & R_{i} .
\end{array}\right)\left(\begin{array}{c}
y \\
\xi
\end{array}\right) \geq-M_{i}
$$

and $Y_{i}<Y_{i+1}$ for $i=\{i=1, \ldots, N-1\}$. Assume that there exist solutions $\left(P_{i}, r_{i}\right)$ to the LMI's

$$
\left[\begin{array}{cc}
A^{T} P_{i}+P_{i} A+C^{T} Q_{i} C+r P_{i} & P_{i} B+C^{T} S_{i} \\
B^{T} P_{i}+S_{i}^{T} C & R_{i}
\end{array}\right]<0
$$

with $P_{i} \in \mathbb{R}^{n \times n}, P_{i}=P_{i}^{T}, P_{i}>0, r_{i} \in \mathbb{R}^{+}$and define

$$
\underline{H_{i}}:=\frac{M_{i}}{r_{i}} \quad \overline{H_{i}}:=\frac{Y_{i}^{2}}{C P_{i}^{-1} C^{T}} .
$$


Then, if $\underline{H}_{i+1} C P_{i+1}^{-1} C^{T}<Y_{i}^{2}$,

$$
\mathcal{E}_{P_{N}}\left(H_{N}\right) \subseteq \mathcal{D}\left(\mathcal{E}_{P_{1}}\left(\underline{H}_{1}\right)\right) .
$$

for any $H_{N}<\bar{H}_{N}$.

Proof: Consider the Lyapunov functions

$$
V_{i}(x)=x^{T} P_{i} x
$$

and compute their derivatives along their trajectories

$$
\begin{aligned}
d V_{i} / d t= & x^{T} A^{T} P_{i} x+x^{T} P_{i} A x+\xi^{T} B^{T} P_{i} x+x^{T} P_{i} B \xi= \\
= & x^{T} A^{T} P_{i} x+x^{T} P_{i} A x+\xi^{T} B^{T} P_{i} x+x^{T} P_{i} B \xi+ \\
& \quad+x^{T} C^{T} Q_{i} C x+2 x^{T} C^{T} S_{i} \xi+\xi^{T} R_{i} \xi-\sigma(y, \xi) \leq \\
\leq & -r_{i} x^{T} P_{i} x-\sigma_{i}(y, \xi)
\end{aligned}
$$

Observe that the hypothesis of Theorem 5.2 are satisfied for any set of parameters with contiguous indexes $(i, i+1)$. Thus, the assertion is proven.

\section{NuMERICAL EXAMPLES}

\section{A. Step by step application of Theorem 5.2}

This example has the objective to exemplify the basic ideas behind Theorem 5.2 following a step by step procedure. We will use the Nyquist plot and the circle criterion as tools to help the intuitive reasoning. Consider a physical system modeled by the transfer function

$$
\begin{aligned}
& \dot{x}=\left(\begin{array}{cc}
0 & -50 \\
1 & 1
\end{array}\right)+\left(\begin{array}{c}
-10 \\
10
\end{array}\right) u \\
& y=\left(\begin{array}{ll}
0 & 1
\end{array}\right) x
\end{aligned}
$$

and assume a saturation on the actuators

$$
u=\operatorname{sat}_{L}(v):=\min \{|v|, L\} \operatorname{sgn}(v)
$$

with saturation level $L=5$ and a nonlinear static controller of the form

$$
v=K_{1} y+K_{3} y^{3} .
$$

with $K_{1}=1.31$ and $K_{3}=3$. A controller as (37) can be useful in situations where it is important to obtain local performances in the neighborhood of the equilibrium $(v \simeq$ $K_{1} y$ ) and at the same time it is desirable to have a prompter response when the system state is far from it. The linear transfer function $G(s)$ is not stable so it is not possible to globally stabilize if because of the input saturation. We want to obtain an estimate of the attraction domain using the criterion proposed in the previous section. The Nyquist plot of (34) is depicted in Figure 4. We can use the circle criterion in order to find an estimate of the domain of attraction. As it is shown in Figure 4 the sector $\mathcal{S}_{1}:=\left(\alpha_{1}, \beta_{1}\right)=(0.17,4.85)$ is a stability sector for the system. It can be verified that $0 \leq K_{3} \leq 3$ implies $n(y):=\operatorname{sat}_{L}(v(y)) \in \mathcal{S}_{1}$ for $|y|<L / \alpha_{1}=$ $Y_{1} \simeq 29.411$. In the BLQC formulation the sector condition of the circle criterion is equivalent to $n(y) \in B L Q C\left(\sigma_{1}, 0\right)$ with multiplier

$$
\Sigma_{1}=\left(\begin{array}{cc}
-\alpha_{1} \beta_{1} & -\left(\alpha_{1}+\beta_{1}\right) \\
-\left(\alpha_{1}+\beta_{1}\right) & -1
\end{array}\right) .
$$

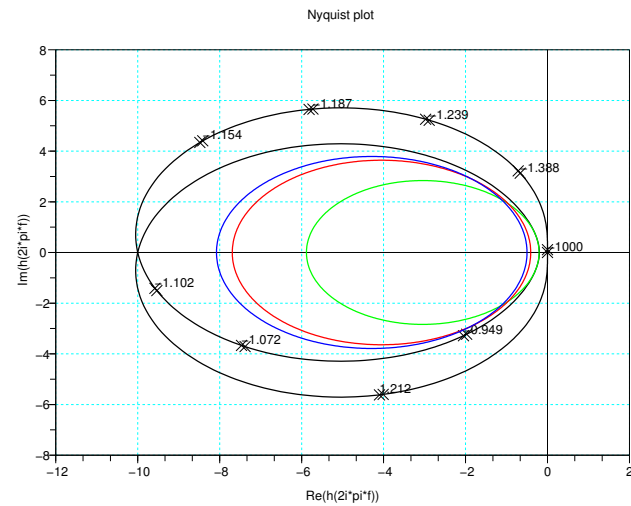

Fig. 4. The Nyquist plot of the linear transfer function (34) (black) and the circles associated to the sectors $S_{1}$ (green), $S_{2}$ (red) and $S_{3}$ (blue) as in example $\mathrm{A}$.

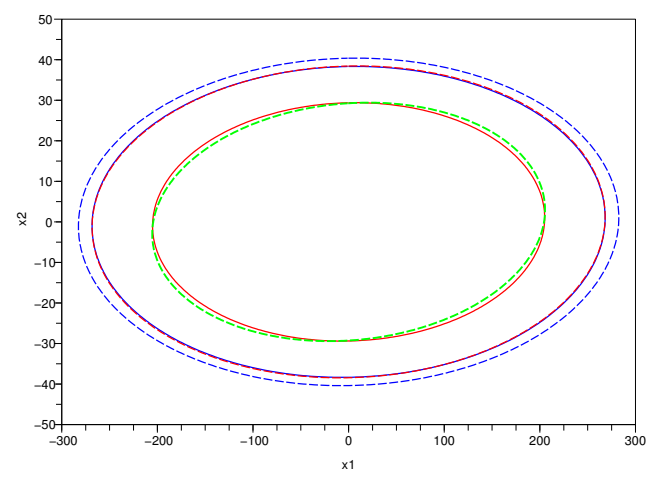

Fig. 5. Attraction Domain Estimates.

A standard way to estimate the domain of attraction is given by the LaSalle theorem exploiting the quadratic Lyapunov function provided by the circle criterion (see the green ellipsoid in Figure 5). It is possible to obtain a better estimate of the attraction domain. We note that $n(y) \in B L Q C\left(\sigma_{2}, M_{2}\right)$ where the BLQC multiplier is

$$
\Sigma_{2}=\left(\begin{array}{cc}
-\alpha_{2} \beta_{2} & -\left(\alpha_{2}+\beta_{2}\right) \\
-\left(\alpha_{2}+\beta_{2}\right) & -1
\end{array}\right)
$$

for $\alpha_{2}=0.13, \beta=2.44, M_{2}=11.7$ and for any $|y|<L / \alpha_{2}=$ : $\bar{Y}_{2} \simeq$ 38.461. Solving the associated LMI's we obtain a Lyapunov function $V_{2}=x^{T} P_{2} x$ which allows to conclude that eventually $|y(t)|<\underline{Y}_{2} \simeq 29.39<\bar{Y}_{1}$ if $x$ belongs to an ellipsoid $\mathcal{E}_{P_{2}}\left(\bar{H}_{2}\right)$. Thus, $\mathcal{E}_{P_{2}}\left(\bar{H}_{2}\right)$ (the larger red ellipsoid in Figure 5) is in the attraction domain. The same procedure can be repeated a third time noting that $n(y) \in B L Q C\left(\sigma_{3}, M_{3}\right)$ for $\alpha_{3}=0.1238, \beta=2.00, M_{2}=13.992$ for $|y|<L / \alpha_{3}=: \bar{Y}_{3} \simeq$ 40.38. In an analogous way we find that $y(t)$ is eventually bounded by $\underline{Y}_{3} \simeq 38.34$. Then the ellipsoid $\mathcal{E}_{P_{3}}\left(\bar{H}_{3}\right)$ (the larger blue ellipsoid in Figure 5) is in the attraction domain. 
B. Contractively invariance vs. combined Lyapunov functions

In [13] a technique to estimate attraction domains is described. Such a technique estimates attraction domains using contractively invariant ellipsoids. The following example demonstrates that the adoption of contractively invariant ellipsoids can be a limiting choice in some situations. Consider the following system (which admits a Lur'e representation)

$$
\begin{aligned}
& \dot{x}=\left(\begin{array}{cc}
-\rho & 1 \\
-1 & -\rho
\end{array}\right)+\left(\begin{array}{c}
-1 \\
0
\end{array}\right) u \\
& y=\left(\begin{array}{ll}
0 & 1
\end{array}\right) x \\
& u=-n(y)=-\frac{y^{2}\left(y^{2}+2\right)}{\left(y^{2}+1\right)^{2}} y
\end{aligned}
$$

with $\rho>0$. Using the Lyapunov function (which is not quadratic)

$$
V(x)=x_{1}^{2}+x_{2}^{2}+\frac{x_{2}^{4}}{x_{2}^{2}+1}
$$

we can prove that the origin of the system is globally asymptotically stable for any positive $\rho$. However, for $\rho<$ $\bar{\rho}_{C I} \simeq 0.204$, it can be proved that there exists a positive value $X_{2}>0$ such that any contractively invariant ellipsoid must lie in the strip $\left|x_{2}\right| \leq X_{2}$. Thus, the approach in [13] can not establish global asymptotical stability. Conversely, we can show that the technique presented in this paper (for $N=2$ ) can prove that the domain of attraction is $\mathfrak{R}^{2}$ even for values of $\rho>\bar{\rho}_{I} \simeq 0.11$.

$$
\text { 1) Computational part: }
$$

Consider Theorem 6.2 with

$$
\begin{gathered}
M_{1}=0, \quad \alpha_{1}=1, \quad \beta_{1}=1.67, \quad Y_{1}=1.4 \\
M_{2}=0.09, \quad \alpha_{2}=1.6, \quad \beta_{2}=2, \quad Y_{2}=+\infty, \\
\Sigma_{i}=\left(\begin{array}{cc}
-\alpha_{i} \beta_{i} & -\left(\alpha_{i}+\beta_{i}\right) \\
-\left(\alpha_{i}+\beta_{i}\right) & -1
\end{array}\right),
\end{gathered}
$$

and solve the associated LMI's. The solutions we found for $\rho=0.11$ using the Scilab Lmi solver are

$$
P_{1}=\left(\begin{array}{ll}
0.2384 & 0.0022 \\
0.0022 & 0.5568
\end{array}\right), P_{2}=\left(\begin{array}{ll}
0.2187 & 0.0084 \\
0.0084 & 0.6131
\end{array}\right) \text {. }
$$

Since the hypothesis are verified, the set $\mathcal{E}_{P_{2}}\left(H_{2}\right)$, for any $H_{2}<\bar{H}_{2}=+\infty$, is in the attraction domain of the attracting set $\mathcal{E}_{P_{1}}(0)=\{0\}$. This is equivalent to global asymptotic stability for the origin.

\section{Conclusions}

In this paper, we have developed a technique to reduce the conservativeness of attraction domain estimates in Lur'e systems. The novelty of our approach lies in the fact that the sets we are considering are invariant but not necessarily contractively invariant as in other criteria. A comparison of our method with other results in the literature is presented using numerical examples.

\section{ACKNOWLEDGMENTS}

The authors are grateful to the developers of Scilab at INRIA. The numerical implementations of the techniques described in this paper have been realized thanks to their valuable work.

\section{REFERENCES}

[1] A. I. Lur'e and V. N. Postnikov, "On the theory of stability of control systems," Prikl. Mat. Mech., vol. 8, pp. 246-248, 1944.

[2] A. Sebastian, M. Salapaka, and D. Chen, "Harmonic and power balance tools for tapping-mode afm," Journal of Applied Physics, vol. 89, pp. 6473-6480, 2001.

[3] A. Sebastian, A. Gannepalli, and M. Salapaka., "A review of the systems approach to the analysis of dynamic-mode atomic force microscopy," IEEE Transactions on Control Systems Technology, vol. 25, pp. 952-959, 2005.

[4] D. Wiesmann and A. Sebastian, "Dynamics of silicon microheaters: Modeling and experimental identification," MEMS 2006,Turkey,Istanbul, p. 044906, 2005.

[5] A. Sebastian and D. Wiesmann, "Modeling and experimental identification of silicon micro-heater dynamics: A systems approach," Proceedings of IFAC World Congress, 2008.

[6] P.Agarwal, D.R.Sahoo, A. Sebastian, H. Pozidis, and M.Salapaka, "Modeling and identification of the dynamics of electrostatically actuated microcantilever with integrated thermal sensor," Proceedings of the Conference on Decision and Control, Dec 2008.

[7] V. M. Popov, "Absolute stability of nonlinear systems of automatic control," Automation and Remote Control, vol. 22, pp. 857-875, 1962.

[8] I. W. Sandberg, "A frequency-domain condition for the stability of feedback systems containing a single time-varying nonlinear element," Bell Syst. Tech. J., vol. 43, pp. 1601-1608, 1964.

[9] G. Zames, "On the input-output stability of time-varying nonlinear feedback systems part two: Conditions involving circles in the frequency plane and sector nonlinearities," IEEE Transactions on Automatic Control, vol. 11, pp. 465 - 476, 1966.

[10] A. Megretski and A. Rantzer, "System analysis via integral quadratic constraints," IEEE Transactions on Automatic Control, vol. 42, pp. 819-830, 1997.

[11] H. K. Khalil, Nonlinear Systems. Upper Saddle River: Prentice-Hall, 1996.

[12] M. Vidyasagar, Nonlinear Systems Analysis. Englewood Cliffs: Prentice Hall, 1993.

[13] T. Hu, B. Huang, and Z. Lin, "Absolute stability with a generalized sector condition," IEEE Trans. Autom. Contr., vol. 49, pp. 535-548, 2004. 